Correction

\title{
Correction: Liu, H.W.; et al. Enhanced Hsa-miR-181d/p-STAT3 and Hsa-miR-181d/p-STAT5A Ratios Mediate the Anticancer Effect of Garcinol in STAT3/5A-Addicted Glioblastoma. Cancers 2019, 11, 1888
}

\author{
Heng-Wei Liu 1,2,3,4, Peter Mingjui Lee ${ }^{5}$, Oluwaseun Adebayo Bamodu 4,6,7@, Yu-Kai Su 1,2,3,4, \\ Iat-Hang Fong ${ }^{3,4}$, Chi-Tai Yeh ${ }^{1,4,6,7,8}$ (), Ming-Hsien Chien ${ }^{1}$, I-Hung Kan ${ }^{1,2,3,4, *}$ \\ and Chien-Min Lin 1,2,3,4,*iD \\ 1 Graduate Institute of Clinical Medicine, College of Medicine, Taipei Medical University, \\ Taipei City 11031, Taiwan; henryway0404@hotmail.com (H.-W.L.); yukai.su@gmail.com (Y.-K.S.); \\ ctyeh@s.tmu.edu.tw (C.-T.Y.); mhchien1976@gmail.com (M.-H.C.) \\ 2 Department of Neurology, School of Medicine, College of Medicine, Taipei Medical University, \\ Taipei City 11031, Taiwan \\ 3 Division of Neurosurgery, Department of Surgery, Taipei Medical University-Shuang Ho Hospital, \\ New Taipei City 23561, Taiwan; impossiblewasnothing@hotmail.com \\ 4 Taipei Neuroscience Institute, Taipei Medical University, Taipei 11031, Taiwan; 16625@s.tmu.edu.tw \\ 5 Department of Clinical Oncology, College of Medicine, California North state University, Elk Grove, \\ California, CA 95757, USA; peter100893@gmail.com \\ 6 Department of Hematology and Oncology, Cancer Center, Taipei Medical University—Shuang Ho Hospital, \\ New Taipei City 235, Taiwan \\ 7 Department of Medical Research and Education, Taipei Medical University-Shuang Ho Hospital, \\ New Taipei City 235, Taiwan \\ 8 Department of Medical Laboratory Science and Biotechnology, Yuanpei University of Medical Technology, \\ Hsinchu City 30015, Taiwan \\ * Correspondence: nskan1999@icloud.com (I.-H.K.); m513092004@tmu.edu.tw (C.-M.L.); \\ Tel.: +886-2-2490088 (ext. 8881) (I.-H.K.)
}

The authors wish to make the following corrections to this paper [1]:

After the publication of this work, we were notified of the mistakes in Figure 1E and Section 3.2, which have now been updated in this correction.

The original Figure $1 \mathrm{E}$ and legend of Figure $1 \mathrm{E}$ are as below: 
E

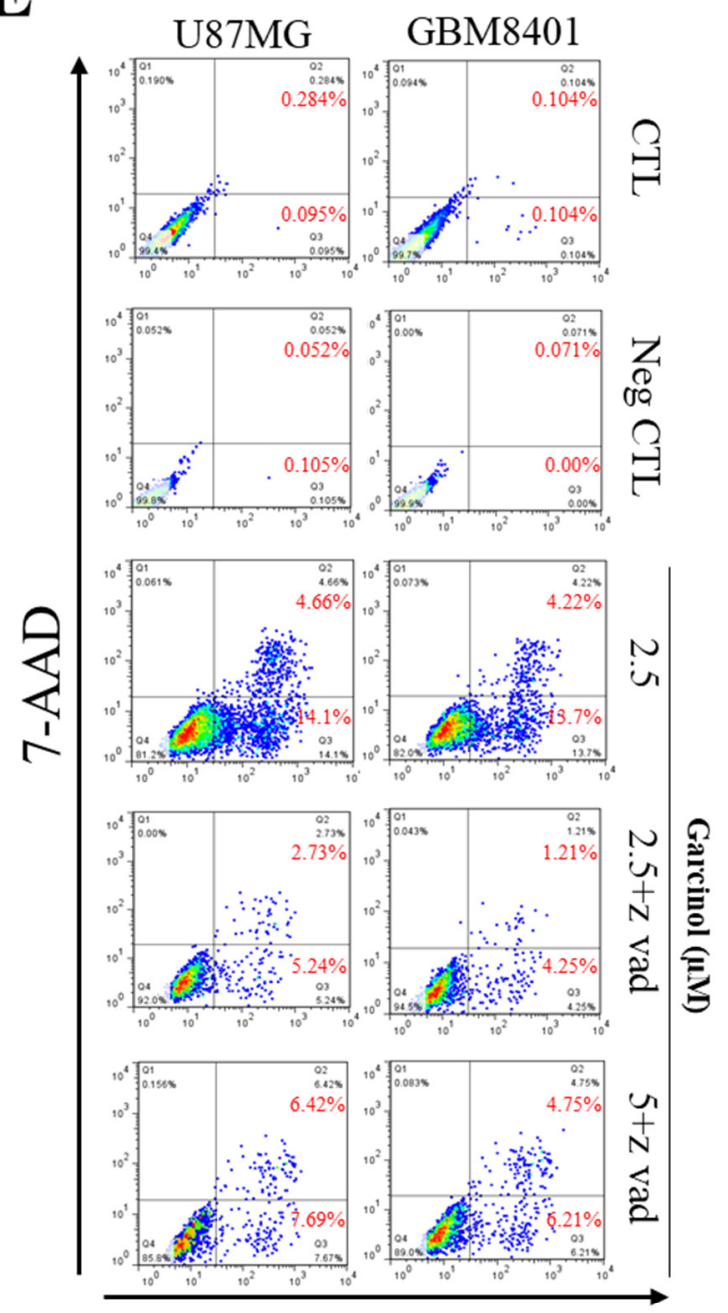

Annexin V

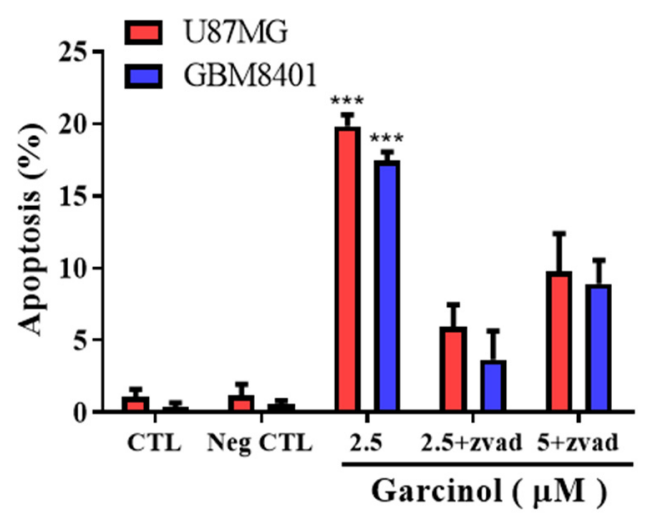

*Neg CTL, caspase inhibitor: Z-VAD-FMK $(20 \mu \mathrm{M})$

Figure 1. (E) Flow-cytometry data (upper) and graphical representation (lower) showing the effect of Garcinol, alone or in presence of Z-VAD-FMK, on U87MG or GBM8401 cells co-stained with PE-conjugated Annexin V and 7-AAD, compared with untreated control or Z-VAD-FMK-treated negative control groups. Annexin V-stained Q4 cells are early apoptotic cells, whereas Q2 cells are late apoptotic (necrotic) cells. Apoptosis (\%), sum of Q4 + Q2; CTL, vehicle-treated; Neg CTL, pan-caspase inhibitor benzyloxycarbonyl-Val-Ala-Asp-fluoromethyl ketone (Z-VAD-FMK). 
and should be replaced with the following:

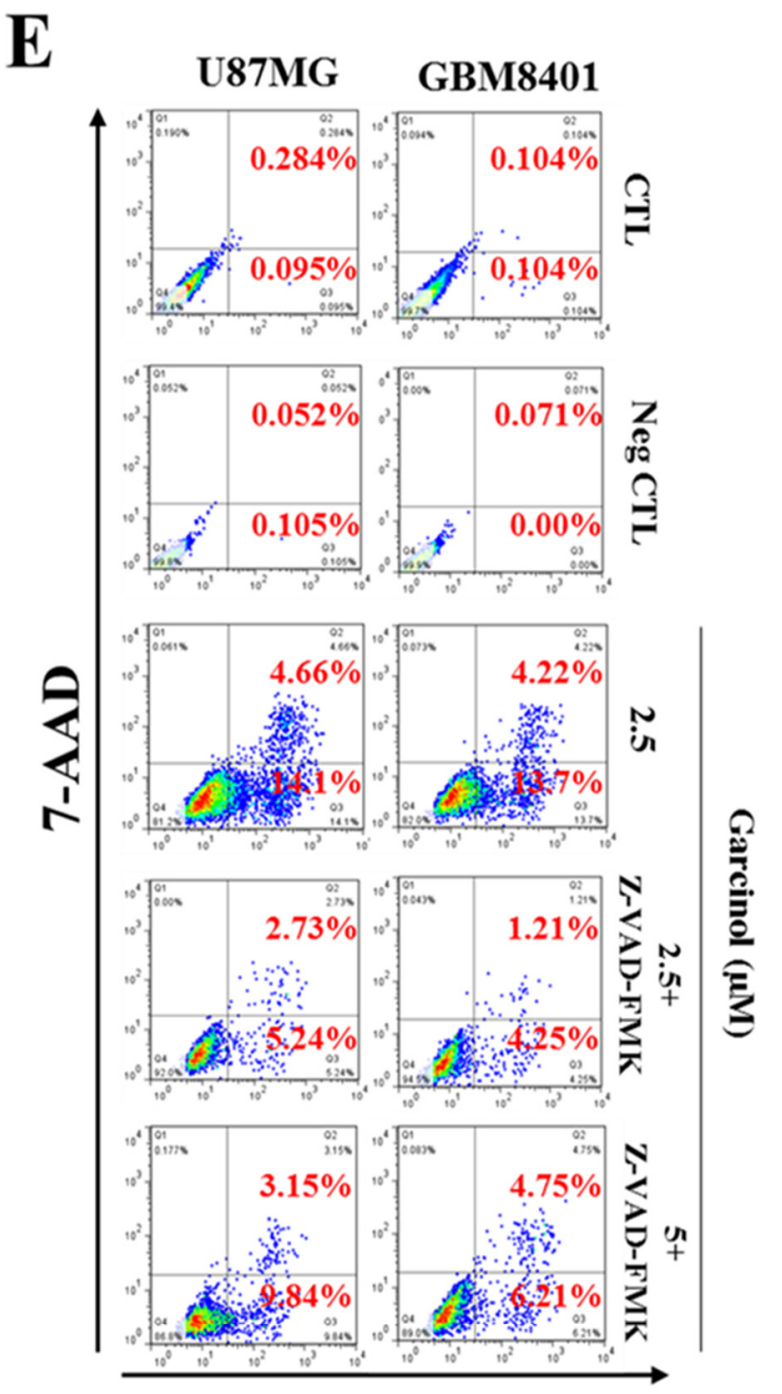

Annexin V

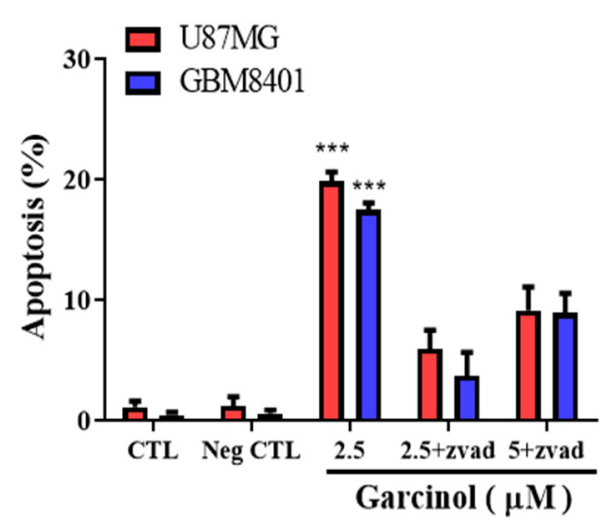

Figure 2. (E) Flow-cytometry data (upper) and graphical representation (lower) showing the effect of garcinol, alone or in presence of Z-VAD-FMK, on U87MG or GBM8401 cells co-stained with PE-conjugated annexin $\mathrm{V}$ and 7-AAD, compared with untreated control or Z-VAD-FMK-treated negative control groups. Annexin V-stained Q3 cells are early apoptotic cells, whereas Q2 cells are late apoptotic (necrotic) cells. Apoptosis (\%), sum of Q3 + Q2; CTL, vehicle-treated; Neg CTL, pan-caspase inhibitor benzyloxycarbonyl-Val-Ala-Asp-fluoromethyl ketone (Z-VAD-FMK). 
In addition, the authors reported errors in Section 3.2 when analyzing the original data in Figure 2E. Thus, the authors wish to replace the following sentence in Section 3.2:

“ $\ldots$ and $7.97 \%$ or $14.11 \%$ apoptosis of the U87MG cells (Figure 1E), indicating that the garcinol-induced cell death was apoptotic and caspase-dependent."

with:

“ $\ldots$ and $7.97 \%$ or $12.99 \%$ apoptosis of the U87MG cells (Figure 2E), indicating that the garcinol-induced cell death was apoptotic and caspase-dependent."

The corrections made in this erratum do not affect the original conclusions. The authors would like to apologize for any inconvenience caused to the readers by these changes.

\section{References}

1. Liu, H.-W.; Lee, P.M.; Bamodu, O.A.; Su, Y.-K.; Fong, I.-H.; Yeh, C.-T.; Chien, M.-H.; Kan, I.-H.; Lin, C.-M. Enhanced Hsa-miR-181d/p-STAT3 and Hsa-miR-181d/p-STAT5A Ratios Mediate the Anticancer Effect of Garcinol in STAT3/5A-Addicted Glioblastoma. Cancers 2019, 11, 1888. [CrossRef] [PubMed]

(C) 2020 by the authors. Licensee MDPI, Basel, Switzerland. This article is an open access article distributed under the terms and conditions of the Creative Commons Attribution (CC BY) license (http://creativecommons.org/licenses/by/4.0/). 